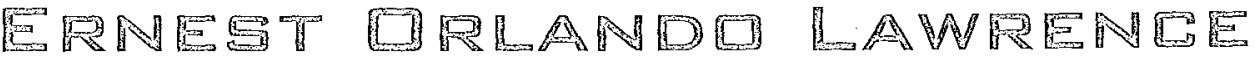

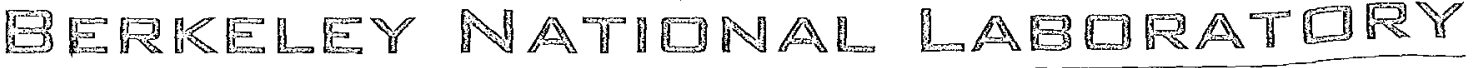

\title{
Seismic Mapping of Subsurface Cavities
}

\author{
Roland Gritto and Ernest L. Majer \\ Earth Sciences Division
}

\author{
November 1999 \\ To be presented at \\ SAGEEP 2000, \\ Arlington, VA, \\ February 20-24, 2000, \\ and to be published in \\ the Proceedings
}

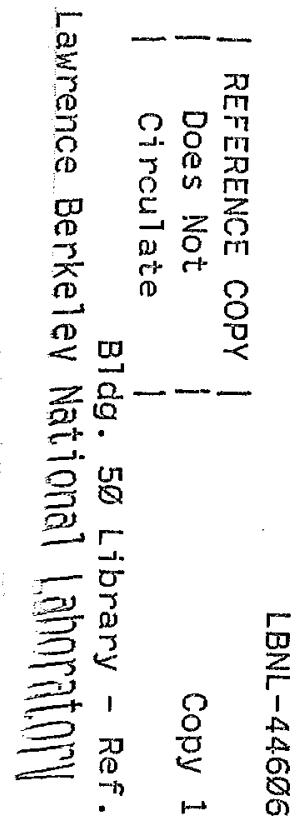


LBNL-44606

\title{
Seismic Mapping of Subsurface Cavities
}

\author{
Roland Gritto and Ernest L. Majer \\ Earth Sciences Division, Lawrence Berkeley National Laboratory, Berkeley, \\ California
}

November 1999

This research was supported by the Director, Office of Science, Division of Basic Energy Sciences, Engineering and Geosciences, of the US Department of Energy under contract No. DE-AC03-76SF00098. Support was also provided by the Defense Special Weapons Agency under Grant DSWA-01-97-1-0026. All computations were carried out at the Center for Computational Seismology of the Lawrence Berkeley National Laboratory. 


\title{
Seismic Mapping of Subsurface Cavities
}

\author{
Roland Gritto and Ernest L. Majer \\ Center for Computational Seismology, Earth Sciences Division \\ Lawrence Berkeley National Laboratory (LBNL) \\ 1 Cyclotron Rd, MS 90-1116 \\ Berkeley, CA 94720
}

\begin{abstract}
This numerical study investigates the possibility of inverting seismic data for the location and volume of a cavity. The data is generated using an exact solution for the scattering of elastic waves by a sphere, whereas the inversion is based on the low frequency Mie approximation to the exact solution. We use correlation analysis between these solutions to solve the inverse problem in two steps. First, the location of the cavity is determined, before the volume is estimated in a second step. The robustness of the results is tested by adding correlated and uncorrelated noise. We find this method to be robust as long as the source receiver coverage is good enough to record various scattered phases (e.g. back and side scattered) and to reduce the level of coherent or random seismic noise. The locations of the cavities are well determined while the estimates of the radii are more susceptible to noise and the deviation between approximation and exact solution.
\end{abstract}

\section{INTRODUCTION}

A problem in geophysical exploration is the determination of the location and the size of cavities in the subsurface. The applications are widespread, ranging from the detection of abandoned mine shafts over construction sites to the detection of underground facilities for military purposes. In the past, attempts have been undertaken to solve this problem by seismic means. Although some methods provide an approximate location of the structure, most fail to give reliable estimates of the actual volume. This is based on the fact that most methods rely on linearized solutions that are not suitable for the problem, as one of their main restrictions is the assumption of small perturbations in the elastic properties. In the case of a cavity, however, the contrast between the surrounding material and the cavity is large, which causes most approximations to fail. Although the strong contrast poses a severe problem, it simultaneously provides valuable apriori information as the elastic parameters of an air filled cavity are known. If through additional measurements (in boreholes or at the surface) the parameters of the surrounding material can be estimated, the experiment reduces to a problem with two unknowns: the location of the center and the volume of the cavity. This scenario is the basis of the current study. 


\section{METHODOLOGY}

In the present case it is assumed that the cavity is located in the subsurface at a depth within reach of a borehole. The geometry for the synthetic experiment is taken from a combination of a reverse VSP and single well survey with sources located in a borehole and receivers located in the borehole and at the surface. Thus backscattered energy is recorded in the borehole while side scattered energy is recorded at the surface. However, sources and receivers are freely exchangeable.

The "field" data is generated using an analytical solution for the scattering of elastic waves by a cavity (Korneev and Johnson, 1996), and using a fixed location and volume of the cavity. The analytic solution contains near and far field terms and is valid for all frequencies. Thus it models the data acquired in the field during a VSP survey. The method used to invert the data is based on the Mie approximation for the scattering of elastic waves by a sphere (Korneev and Johnson, 1993) which is an approximation to the exact solution in the low to intermediate frequency range. It offers the advantage of fast computational speed.

The geometry of the numerical experiment consists of a $20 \mathrm{~m}$ deep borehole containing 21 equally spaced sources and 11 receivers, while 5 additional receivers are located at the surface extending away from the borehole (Figure 1b). To generate the initial data, a cavity with a chosen radius is fixed in space and the scattered wavefield is computed based on the analytic solution. To estimate the location of the cavity, the search area is subdivided into 200 grid points $(10 \times 20)$ and the scattered wavefield based on the Mie approximation is subsequently computed for a cavity of fixed radius at each point. The scattered wavefield is computed for a total of 22 frequencies ranging from $100-1000 \mathrm{~Hz}$. After the generation of the wavefields for all 200 possible cavity locations, a correlation coefficient is computed between the wavefields based on the exact and the approximate solution for each grid point. The correlation coefficient is defined as,

$$
\rho_{e a}=\frac{U_{e} U_{a}^{*}}{\sqrt{U_{e} U_{e}^{*}} \sqrt{U_{a} U_{a}^{*}}}
$$

where Ue and Ua are the wavefields computed using the exact solution and the Mie approximation, respectively, while $*$ denotes the complex conjugate. At each grid point this coefficient is computed for all source receiver combinations and stacked over all frequencies. A map of the correlation coefficients shows the most likely location of the cavity (see Figure $1 b$ ). In a second step, the location of the cavity is fixed at the point of highest correlation, and its radius is varied between $\mathrm{R}=0.1-5.0 \mathrm{~m}$. 
For each value of $\mathrm{R}$ the correlation between exact solution and approximation is computed for all source receiver combinations and stacked over all frequencies again, with the highest correlation coefficient indicating the best estimate of the cavity radius. The correlation coefficient is given by

$$
r_{e a}=-e^{-\frac{\left(U_{e}-U_{a}\right)^{2}}{2 \sigma^{2}}}
$$

where $\sigma$ is the variance which is set to 1 in our case.

In repeated experiments, uncorrelated Gaussian noise is added to the data to test the robustness of the inversion and source receiver geometry, while correlated noise is modeled by the presence of two cavities with different volumes.

\section{RESULTS}

Figure 1 shows the results of the first experiment. The geometry is detailed in Figure 1b. The distribution of sources (stars) and receivers (triangles) can be seen along the border of the model. A single cavity with a radius of $\mathrm{R}=1.2 \mathrm{~m}$ is located at $\mathrm{x}=5.5 \mathrm{~m}$ and $\mathrm{z}=7.5 \mathrm{~m}$ (indicated by the circle). The elastic parameters of the background medium are $V p=5.3 \mathrm{Km} / \mathrm{s}, V s=3.2 \mathrm{Km} / \mathrm{s}$, and $\rho=2.65 \mathrm{~g} / \mathrm{ccm}$. The scattered field based on the analytic solution is given in Figure 1a, while the result of the correlation analysis for the location of the cavity is indicated in Figure $1 \mathrm{~b}$ by the shading and the contour plot. It can be seen that the highest correlation (0.9) plots at the center of the cavity. It is also apparent that the correlation coefficient decreases gradually with increasing distance from the center of the cavity. The pattern is not symmetric, however, but governed by the source-receiver geometry. The results of the second inversion step are presented in Figure 1c. The correlation coefficient is shown as a function of radius. The dashed line represents the actual value $\mathrm{R}=1.2 \mathrm{~m}$ used for the forward modeling. It can be seen that the estimate for the radius slightly underestimates the true radius with a value of $\mathrm{R}=1.1 \mathrm{~m}$. This underestimation is based on the fact that the Mie approximation slightly overestimates the amplitudes of the exact solution at higher frequencies which translates into a smaller volume for equal amplitudes.

In a second step, the first experiment is repeated with an increased noise level to test the robustness of the correlation analysis. In this case, 500\% of the RMS amplitude of the seismic signal is used as uncorrelated Gaussian noise which is added to the scattered data computed with the exact analytical solution. The data are shown in Figure 2a. It can be seen that the phases visible in Figure 1a have disappeared below the noise level. Referring to Figure $2 b$, however, it becomes evident that the highest correlation coefficient still coincides with the center of the sphere, although its value has dropped to a maximum of 0.1 . This surprising result can be explained by 
the stacking technique which incorporates the results of 21 sources, 11 receivers, and 22 frequencies. It reduces the noise level effectively such that a correlation analysis is still viable. The estimate of the radius is given in Figure 2c. It can be seen that the curve developed a broader maximum, although the peak value is still at $\mathrm{R}=1.1 \mathrm{~m}$.

Although a noise level of 500\% may seem high, the fact that the noise is uncorrelated helps to achieve good results during the analysis. In the next step, it is investigated how correlated noise affects the inversion procedure. Therefore, the experiment is repeated using two cavities with different size assuming that the respective scattered wavefields will produce correlated noise for each other during the inversion process. The locations of the two cavities are given in Figure $3 \mathrm{~b}$ (indicated by the circles). Their radii are $\mathrm{R}=1.4 \mathrm{~m}$ and $\mathrm{R}=0.8 \mathrm{~m}$. The scattered wavefields seen in Figure $3 \mathrm{a}$ indicate a more complicated pattern. The inversion for the locations, however, produces similar promising results. It can be seen that the larger cavity is correctly located with a correlation coefficient of 0.8 , while for the smaller cavity a drop in correlation coefficient to 0.3 is visible at the right location. Considering the pattern of the correlation coefficient around the larger cavity which acts as noise for the smaller one, it may be stated that the two cavities should have a separation of one cavity diameter for a successful estimation of their locations. Referring to the inversion for the radii (Figure 3c), it can be seen that similar estimates are achieved in both cases. The perfect estimate for the smaller cavity may be accidentally caused by the noise of the larger one, as both radii should be underestimated according to the argument made for the misfit between the two solutions at higher frequencies.

However, the overall result is very encouraging such that the last experiment will test the inversion process for two cavities in the presence of uncorrelated noise, while the background velocities are overestimated by $10 \%$. The results of this test are given in Figure 4. The locations and radii are the same as before, while the level of uncorrelated Gaussian noise is kept at 300\%. This produces scattered wavefields without apparent coherent phases Figure 4a). The inversion result for the locations is given in Figure $4 \mathrm{~b}$. It can be seen that the largest correlation coefficients shifted slightly from the center of the cavities away from sources and receivers which is caused by overestimating the background velocity. Incorrect velocity estimates will shift the phases farther from, or closer to, sources and receivers for higher or lower velocity values, respectively. Therefore, the estimate of the location of the cavity looses accuracy. Furthermore, the Gaussian noise reduced the correlation coefficients to 0.15 and 0.05 for the large and small cavity, respectively. The estimates of the radii show a similar pattern (Figure $4 \mathrm{c}$ ). The smaller radius is overestimated with a value of $0.9 \mathrm{~m}$ while the larger one is underestimated with a value of $1.3 \mathrm{~m}$.

Considering the complications added to this numerical experiment (the presence of correlated and uncorrelated noise and overestimating the background velocities) the results suggest that the presented inversion method is robust enough to solve the problem of cavity detection in real field environments. 


\section{CONCLUSION}

The presented two step inversion to solve for the location and the size of an underground cavity appears to be a fast and robust method to determine these parameters in field situations. The Mie approximation can be applied whenever a spherically shaped cavity is the target of the investigation.

Knowing the elastic parameters a priori reduces the problem to a 2 parameter estimation for the location of the center and the radius. Therefore, a detailed search using a fast approximation is a feasible approach to this problem. The correct phase information provides the location of the cavity and therefore is susceptible to variations in the background velocity. The radius of the cavity is determined by the amplitudes of the scattered wavefield, and therefore is affected by data noise, even if the noise is uncorrelated. However, a carefully selected source receiver geometry which provides enough coverage to record back and side scattered phases and which is simultaneously capable to reduce uncorrelated noise, can be effectively used even under extreme noise conditions.

\section{ACKNOWLEDGEMENTS}

This research was supported by the Director, Office of Science, Division of Basic Energy Sciences, Engineering and Geosciences, of the US Department of Energy under contract DE-AC03-76SF00098. Support was also provided by the Defense Special Weapons Agency under grant DSWA-01-97-1-0026. All computations were carried out at the Center for Computational Seismology of the Lawrence Berkeley National Laboratory.

\section{REFERENCES}

Korneev V.A. and Johnson L.R., 1993, Scattering of elastic waves by a spherical inclusion - II. Limitations of asymptotic solutions, Geophys. J. Int., Vol. 115, pp. 251263.

Korneev V.A. and Johnson L.R., 1996, Scattering of P and S waves by a spherically symmetric inclusion, PAGEOPH, Vol. 147, pp. 675-718. 


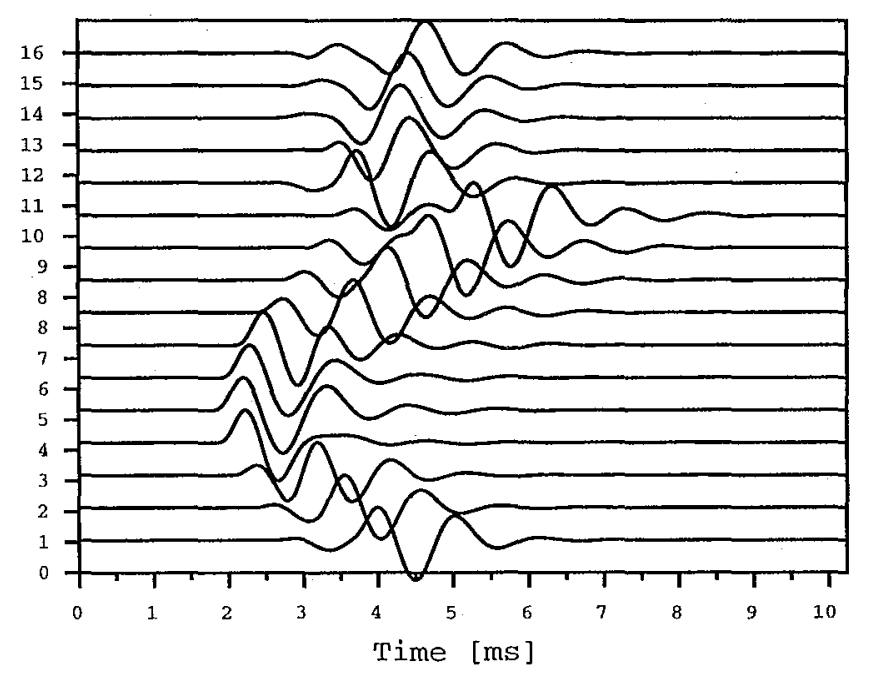

Figure 1a: Exact scattered field for geometry given in Figure 1b. First 10 traces are recorded in the borehole, while last 6 are recorded at the surface.

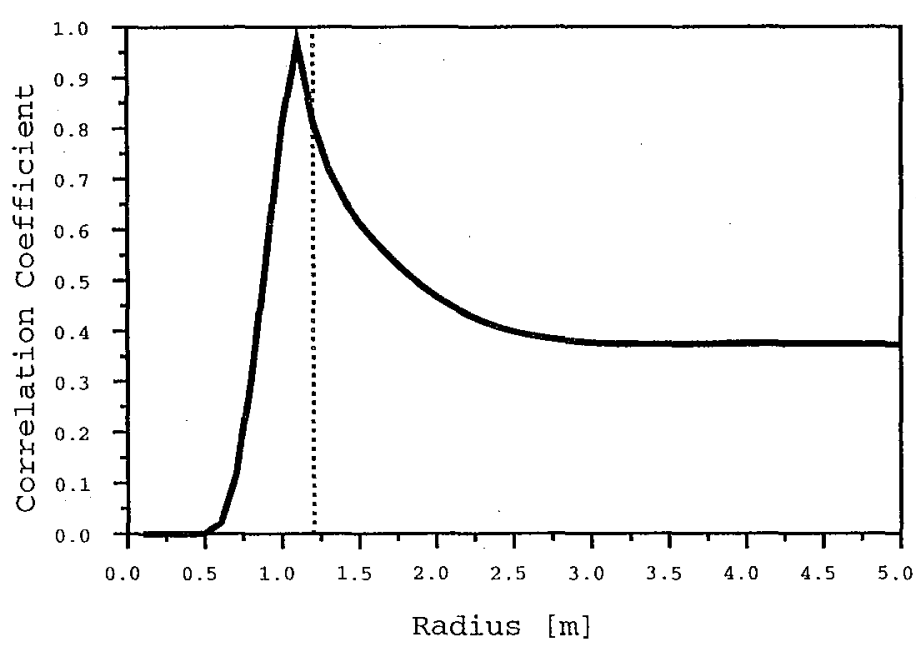

Figure 1c: Result of the second inversion step. The original radius is indicated by the dashed line, while the correlation values are given by the solid line.

\section{Correlation Map, $0 \%$ Noise}

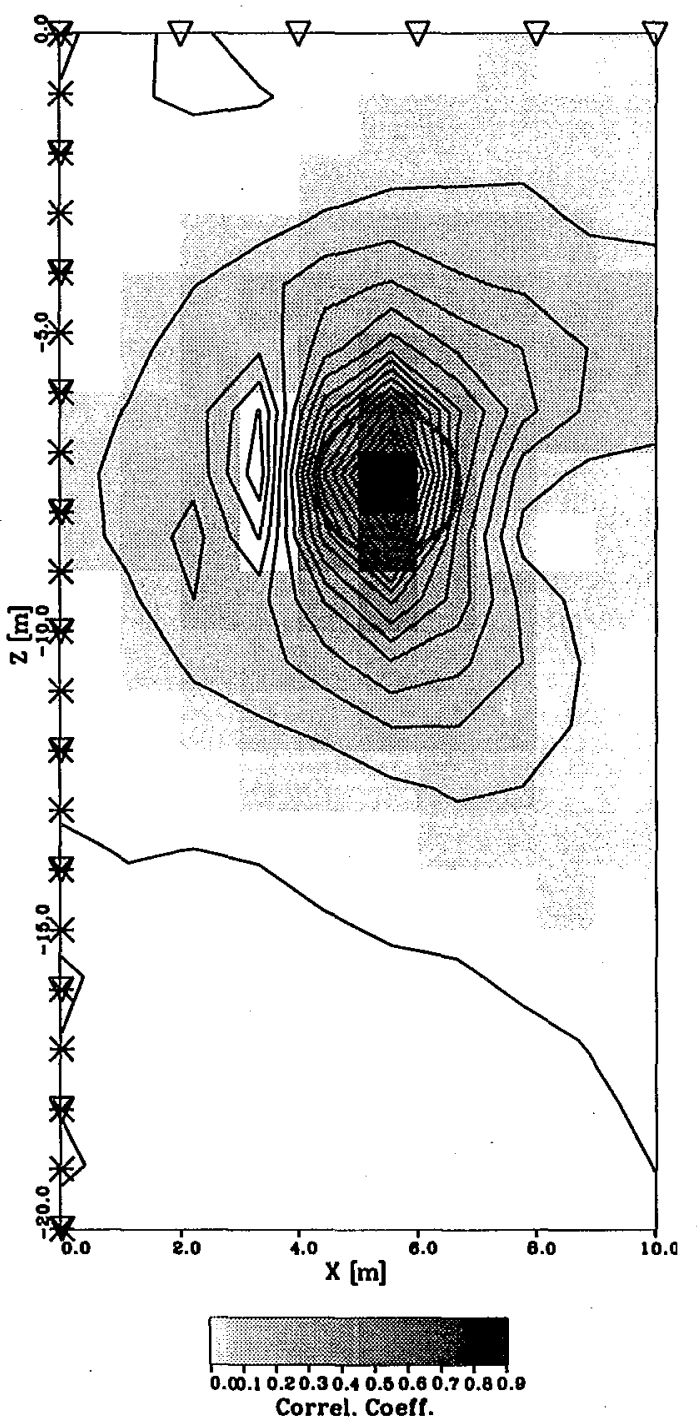

Figure 1b: Geometry of the experiment. 21 sources (stars) and 11 receivers (triangles) are equally spaced in a borehole between $0 \mathrm{~m}$ and $20 \mathrm{~m}$, while 6 receivers (triangles) are located along the surface. The circle indicates the location of the original cavity. Shading and contour lines represent the results of the correlation analysis to locate the center of the cavity. 


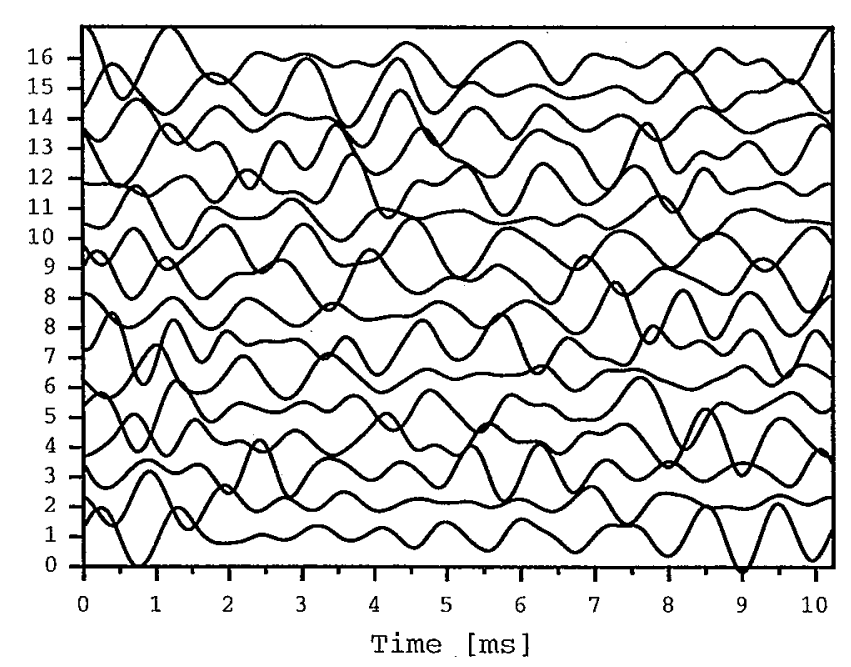

Figure 2a: Same as data in Figure 1a, including $500 \%$ uncorrelated Gaussian noise.

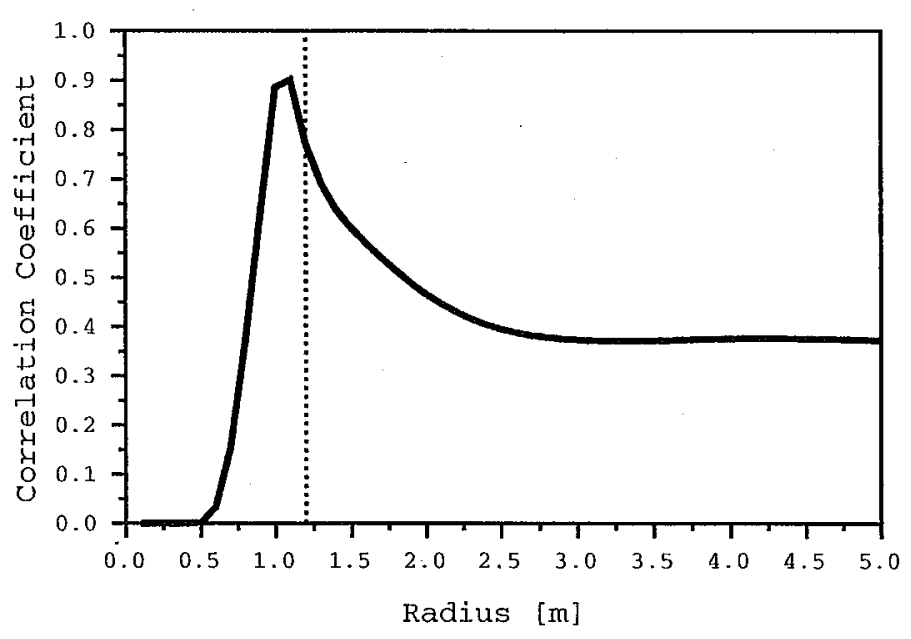

Figure 2c: Same as Figure 1c, including 500\% uncorrelated Gaussian noise.
Correlation Map, 500\% Noise

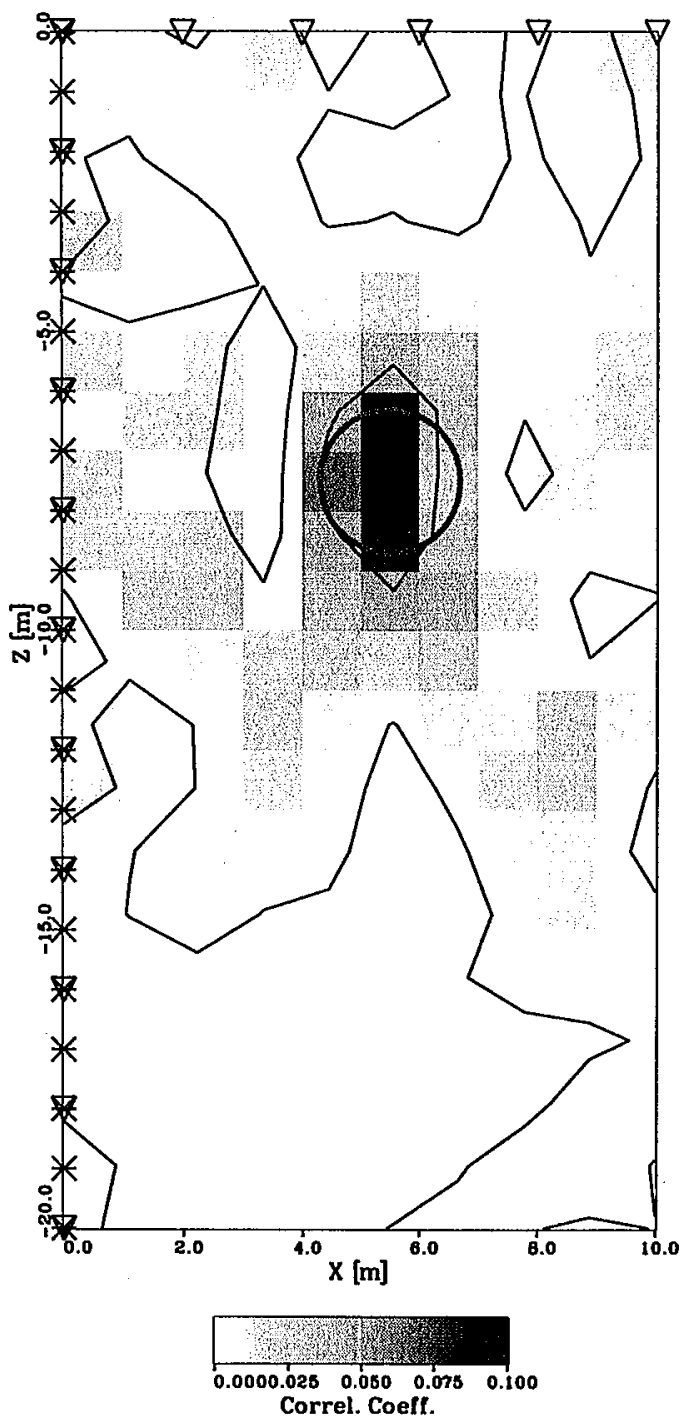

Figure 2b: Same as Figure1b, including 500\% uncorrelated Gaussian noise. 


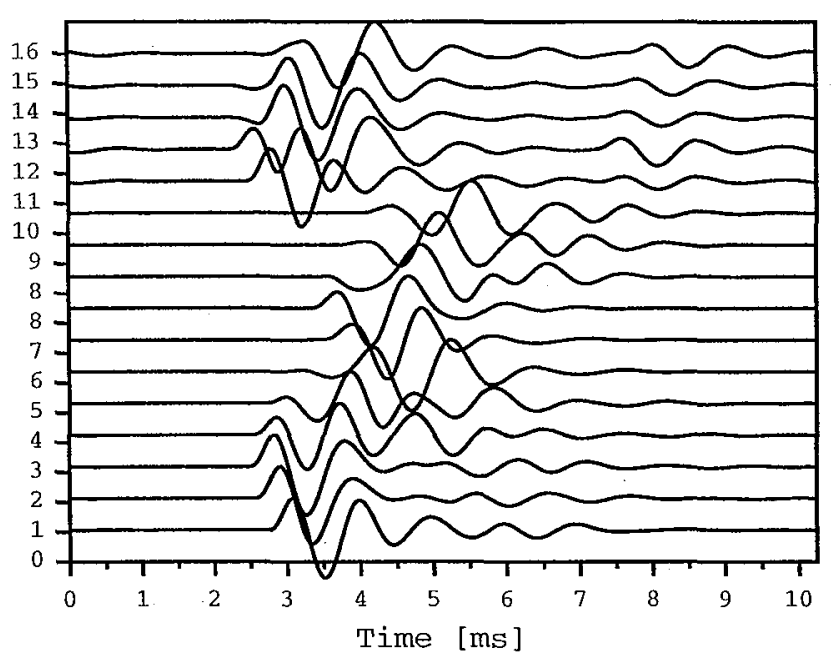

Figure 3a: Same as data in Figure 1a, generated in the presence of two cavities, see Figure $3 b$.

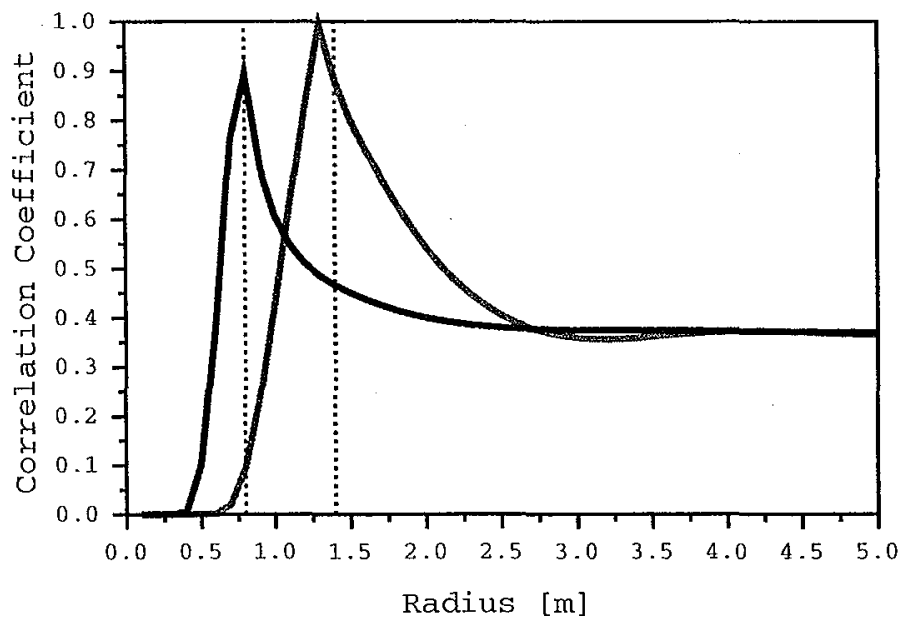

Figure 3c: Same as Figure 1c, for 2 cavities of radius $R=0.8 \mathrm{~m}$ and $\mathrm{R}=1.4 \mathrm{~m}$.
Correlation Map, 0\% Noise

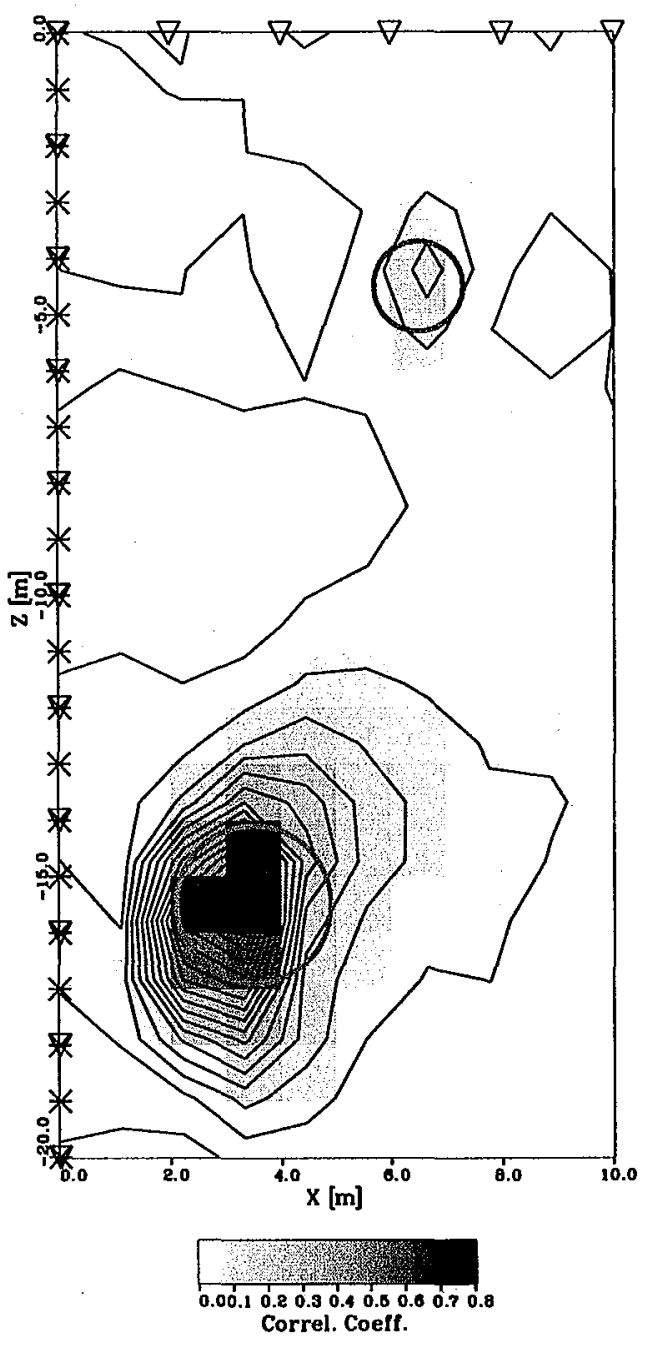

Figure 3b: Same as Figure $1 b$, for 2 cavities with locations indicated by the circles. 


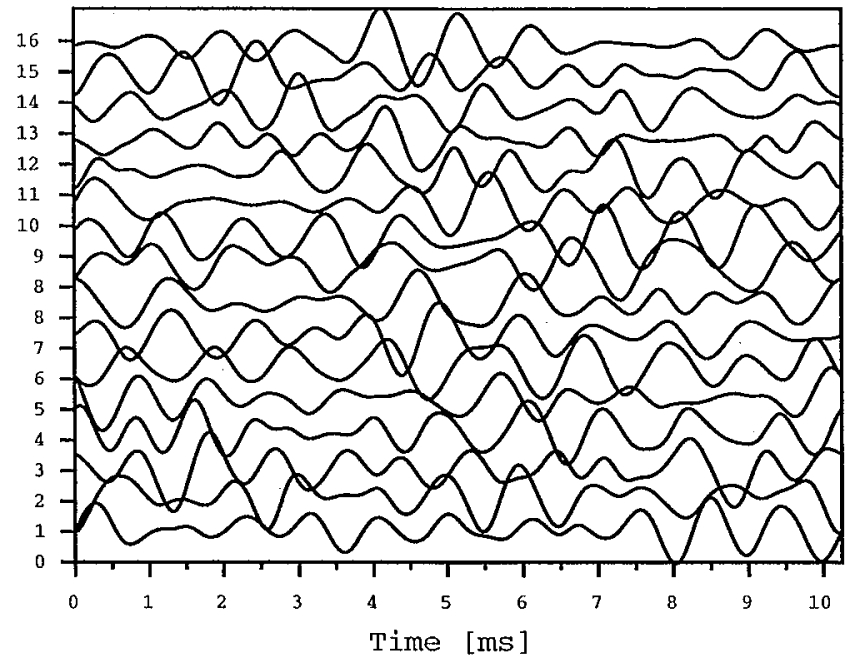

Figure 4a: Same as data in Figure 1a, generated in the presence of two cavities, with the addition of $300 \%$ uncorrelated Gaussian noise.

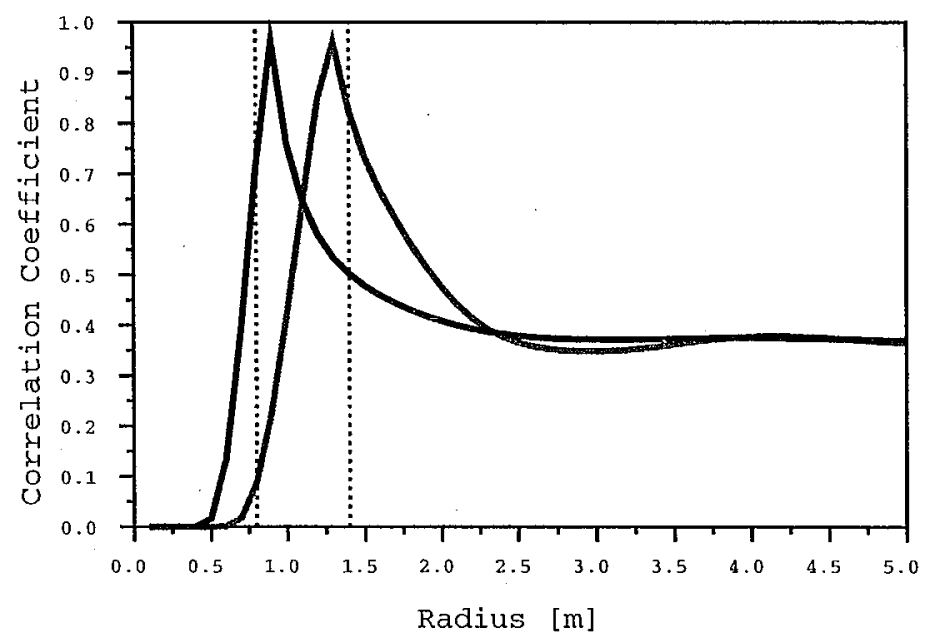

Figure 4c: Same as Figure 1c, for 2 cavities of radius $R=0.8 \mathrm{~m}$ and $R=1.4 \mathrm{~m}$ and $300 \%$ uncorrelated Gaussian noise. During the inversion the background velocity was overestimated by $10 \%$.
Correlation Map, Vel $=+10 \%, 300 \%$ Noise

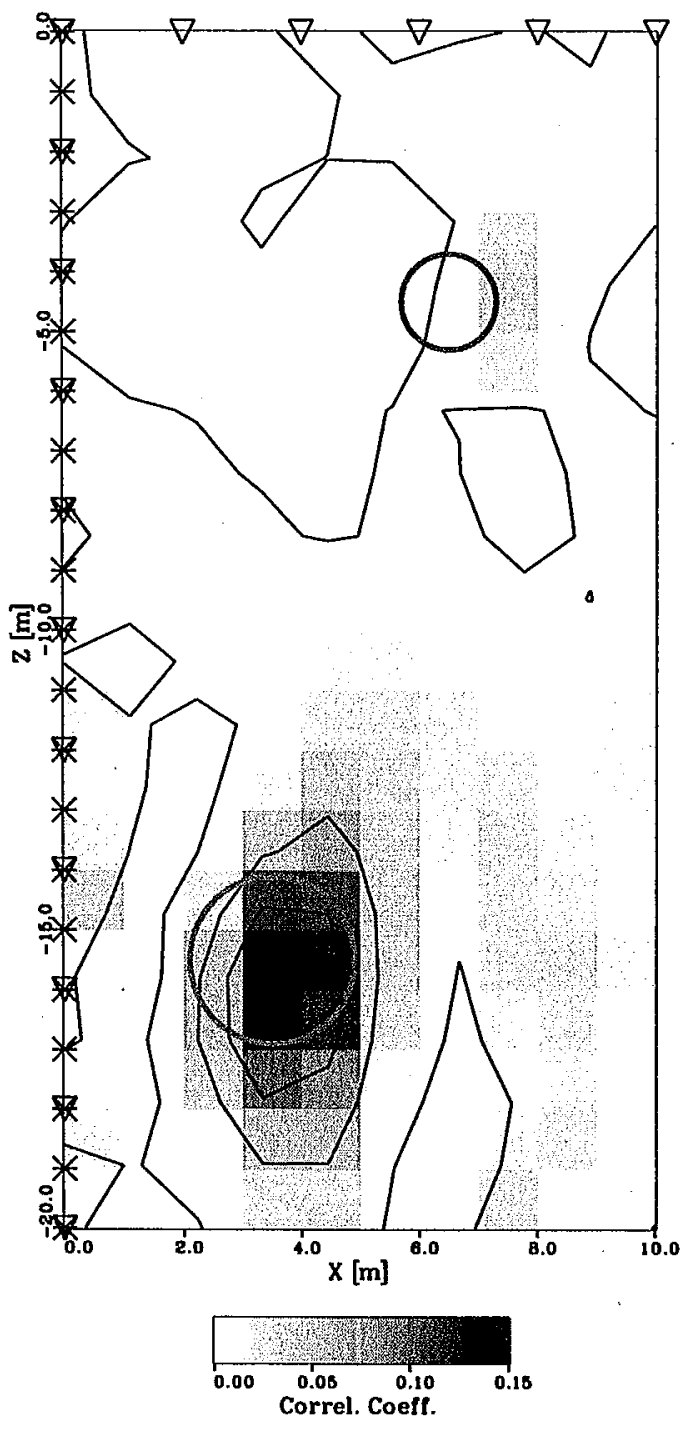

Figure 4b: Same as Figure1b, for 2 cavities with locations indicated by the circles, including $300 \%$ uncorrelated Gaussian noise. During the inversion the background velocity was overestimated by $10 \%$. 


\section{DISCLAIMER}

This document was prepared as an account of work sponsored by the United States Government. While this document is believed to contain correct information, neither the United States Government nor any agency thereof, nor The Regents of the University of California, nor any of their employees, makes any warranty, express or implied, or assumes any legal responsibility for the accuracy, completeness, or usefulness of any information, apparatus, product, or process disclosed, or represents that its use would not infringe privately owned rights. Reference herein to any specific commercial product, process, or service by its trade name, trademark, manufacturer, or otherwise, does not necessarily constitute or imply its endorsement, recommendation, or favoring by the United States Government or any agency thereof, or The Regents of the University of California. The views and opinions of authors expressed herein do not necessarily state or reflect those of the United States Government or any agency thereof, or The Regents of the University of California. 